\title{
TEEN Protocol in Wireless Sensor Network
}

\author{
Ajit K. Bhovi and Dr.Gopal A. Bidkar
}

\begin{abstract}
Network is an interconnection between computers using wired or wireless medium. In wireless, major difficulty faced by the system is transmission of data from one system (node) to another system (node) as compared to wired. In recent years many researchers are working towards data transmission in two major areas of wireless network: one is Wireless AdHoc Network and another is Wireless Sensor Network (WSN). Another and the main problem of WSN is Network lifetime (i.e. how long sensor nodes can survive on battery which are embedded in them). So to overcome these two problems researchers have proposed many protocols and they succeeded upto some extent. In this paper Thresholdsensitive Energy Efficient Network (TEEN) protocol discussed and implemented and then graph is drawn with respect Number of dead nodes Vs Number of rounds, where in every round, head nodes are getting change after some interval of time (Cluster Period).
\end{abstract}

Keywords--- Hard Threshold (HT), Soft Threshold (ST), Sensed Value (SV), Current Value (CV)

\section{INTRODUCTION}

$\mathrm{N}$ ETWORK in broader aspect is a group of people or things. With respect to technical terms network is a interconnection between computers, connections may be wired or wireless. The evolution of network has started with very small group of computers and now it is extended upto group of intelligent computers (sensors) communicating with each other by means of wireless medium, this type of network is called as Wireless Sensor Network (WSN). Wireless Sensor Network (WSN) consist of sensor nodes placed randomly or in pertcular fashion, which have the capability to sense the environment, process the sensed data and send the sensed data to the base station. WSN consist of four units sensing unit, processing unit, transceiver unit and power unit as shown in figure 1 [1]. The sensor network can be used in various applications like health monitoring, environmental parameter monitoring, battle field monitoring, and industrial application, tracking the parameters, home application and mainly military application. WSN are low power (operated by battery), multifunctional, small in size and communicate for a small distance.

In WSN nodes can be deployed in a randomlly or manually depends upon user requirements. Each node has a capability to communicate with the other node or directly to the sink node.One of the major challenges in WSN is energy

Ajit K. Bhovi, Assistant Professsor, Department of ECE, KLS's Vishwanathrao Deshpande Rural Institute of Technology, Haliyal, India. E-mail:ajitbhovi@gmail.com

Gopal A. Bidkar, Professor, Department of ECE, Shri Dharmastala Manjunatheshwar College of Engineering and Technology, India. E-mail: gabidkar@rediffmail.com

DOI: 10.9756/BIJRCE.8188 or power constraint regarding power resources and computational capacity.

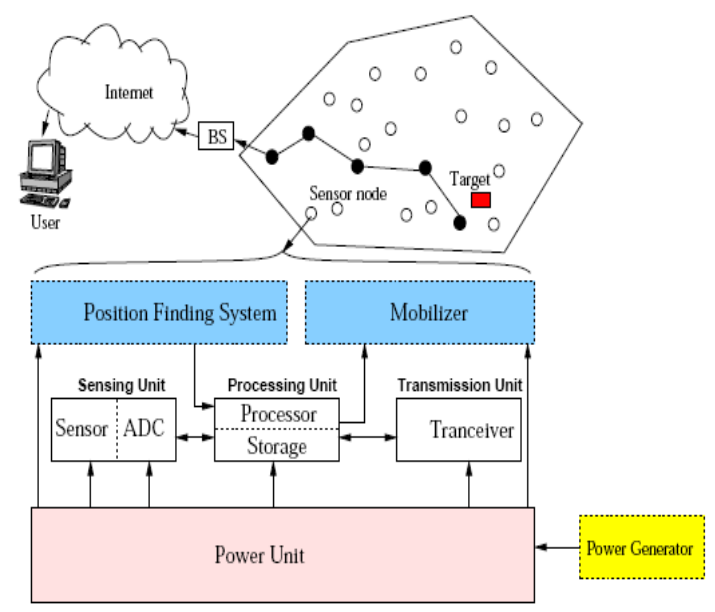

Figure 1: Architecture of WSN

Protocols are designed to overcome the challenges in WSN; protocol is a set of rules defined to do some work. WSN protocols are designed with many objectives; in all these objectives the main objective is to transmit the data from source to destiny in minimum time and with less amount of energy consumption because all the sensor nodes are using a constant DC supply (i.e. battery). To minimize the consumption of energy the protocols should be designed in such a way that it should take minimum cost path between source and destiny and another important is aggregating the data in one node before transmitting to next node. Aggregation is a method in which data from different nodes are collected and added or averaged in one node and transmit, by doing this the data flow in the network is reduced, in other word less amount of energy can be consumed.

Routing Algorithms [1] can be proactive, reactive, centralized, distributed, broadcast, etc. Routing means transferring the data from target to sink. The authors proposed different kinds of protocols [2], [3], [4], [5] in this field.

The issues of WSN are explained in [2]. WSN can be used to find temperature, humidity, pressure, fire detection and multimedia data like image, video and audio. Routing in WSN is a major issue as all the nodes are are same and operated on battery (DC supply). By sending more number datas through the same path we may end up with dead node as battery gets drain quickly.

Data aggregation has been put forward as an essential paradigm for wireless routing in sensor networks. The idea is to combine the data coming from different sources enroute eliminating redundancy, minimizing the number of transmissions and thus saving energy. This paradigm shifts the focus from the traditional address-centric approaches for 
networking (finding short routes between pairs of addressable end-nodes) to a more data-centric approach (finding routes from multiple sources to a single destination that allows innetwork consolidation of redundant data). There are many types of aggregation techniques in WSN namely centralized approach, In-network aggregation [6], Tree-based approach [7] and Cluster based approach [8] etc.

In this paper, TEEN routing protocol is studied and implemented and then graph is drawn with respect to Network lifetime, Number of alive nodes and Number of dead nodes Vs Number of rounds, where in every round, head nodes are getting change after some interval of time.

The rest of the paper is organized as: section II contains the proposed work, section III describes results and section IV describes about conclusion.

\section{PROPOSED WORK}

In this paper the TEEN protocol [9] is implemented, which is a reactive network protocol. This protocol organizes the sensor nodes into multiple levels of hierarchy as shown in figure 2., where data's of all sensor nodes are aggregated and transmitted by the cluster heads until it reaches the base station; cluster heads are chosen depends on their remaining energy. Here two data thresholds are considered in order to minimize the number of transmissions and increase the network lifetime.



Figure 2: Hierarchical Clustering in TEEN

The two thresholds are Hard Threshold (HT) and Soft Threshold (ST).

1. Hard Threshold (HT): This is a threshold value for the sensed attribute. It is the absolute value of the attribute beyond which, the node sensing this value must switch on its transmitter and report to its cluster head.

2. Soft Threshold (ST): This is a small change in the value of the sensed attribute which triggers the node to switch on its transmitter and transmit.

\section{A. Explanation of Protocol}

All the nodes sense their environment continuously. At the first cluster period (round), if data reaches its HT value, the node switches on its transmitter and sends the SV and this SV is also stored in the internal memory of the sensor node. After this cluster period (i.e. in up coming cluster period) all the nodes start transmitting the data, only when both the following conditions are true:

1. If the CV of the sensed data is greater than the HT.

2. If the CV of the sensed data differs from SV by an amount equal to or greater than the ST.

Whenever a sensor node transmits data, SV is set to CV. Thus, the HT tries to reduce the number of transmissions by allowing the nodes to transmit only when the sensed data is in the range of interest, the ST further reduces the number of transmissions by eliminating all the transmissions which might occur when there is little or no change in the sensed data.

\section{RESULTS}

In this section, we present the results which are simulated using MATLAB. In the simulation we considered 100 sensor nodes which are deployed randomly in $400 * 400$ area network. We present the results by considering the parameters like number of dead nodes/round and the network life time is analysed with respect to number of dead nodes for every round or cluster period.

\section{A. Network Lifetime}

The life time of the sensor node mainly depends on the battery life time and its power. Sensors nodes will be active or alive whenever the sensor nodes have the information otherwise nodes will be inactive or sleep mode. In active mode sensor nodes consume more power and in inactive mode nodes consumes less power. For each of the simulation, sensor node sends the varying number of data. In our work we are considering the initial battery of sensor nodes is 0.5 Joules. As we stated above for each data transfer and data aggregation the sensor node looses 50nJ and 5nJ energy respectively. At some point of time the battery gets drained and there will be no energy in the battery to transfer and aggregate the data then node is considered as dead node. From figure 3 we can notice that the total number of dead nodes increases as the number of rounds increases.

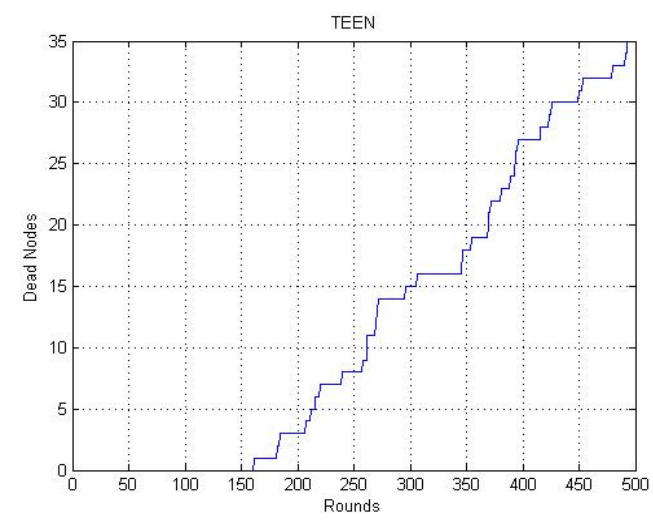

Figure 3: Dead Nodes v/s Number of Rounds

\section{CONCLUSION}

Many researches are going on routing protocol in sensor network. All encountering same issue (i.e. network lifetime), 
so all protocol main aim is to increase this issue. In this paper, we have introduced a network protocol, which is a heirarchial based protocol (i.e. TEEN). In this we got the result which are very satisfactory. As we can see that the count of the dead nodes will starts after 150 rounds and upto 500 rounds only 35 nodes are dead. This shows that there are still good number of nodes which are participating in transmission and the whole network is still alive. In future we would like implement APTEEN protocol and compare with this protocol.

\section{REFERENCES}

[1] I.F. Akyildiz, W. Su, Y. Sankarasubramaniam and E. Cayirci, "A survey on sensor networks", IEEE communications magazine, Vol.40, No.8, Pp.102-114, 2002.

[2] I.F. Akyildiz and X. Wang, "A Survey on Wireless Mesh Networks", IEEE Radio Communications,Vol.43, No.9, Pp.S23-S30, 2005.

[3] J.N. Al-Karaki and A.E. Kamal, "Routing techniques in wireless sensor networks: a survey", IEEE wireless communications, Vol.11, No.6, Pp.6-28, 2004.

[4] W. Heinzelman, A. Chandrakasan and H. Balakrishnan, "EnergyEfficient Communication Protocol for Wireless Micro-sensor Networks", Proceedings of the 33rd Hawaii International Conference on System Sciences, 2000.

[5] S. Lindsey and C.S. Raghavendra, "PEGASIS: Power-efficient gathering in sensor information systems", In Aerospace conference proceedings. Vol.3, Pp.3-1125, 2002.

[6] E. Fasolo, M. Rossi, J. Widmer and M. Zorzi, "In-network aggregation techniques for wireless sensor networks: a survey”, IEEE Wireless Communications, Vol.14, No.2, Pp.70-87, 2007.

[7] M. Lee and V.W.S. Wong, "An Energy-aware Spanning Tree Algorithm for Data Aggregation in Wireless Sensor Networks", IEEE PacRrim 2005, Victoria, BC, Canada, Pp.300-303, 2005.

[8] K. Dasgupta, K. Kalpakis and P. Namjoshi, "An Efficient Clustering based Heuristic for Data Gathering and Aggregation in Sensor Networks”, Vol.42, No.6, Pp.697-716, 2003.

[9] A. Manjeshwar and D.P. Agrawal, "TEEN: ARouting Protocol for Enhanced Efficiency in Wireless Sensor Networks”, IPDPS, Vol.1, Pp.189, 2001. 